\title{
LA DETTE DANS LES RAPPORTS DE GÉNÉRATIONS : UNE TRANSMISSION EN CHANGEMENT
}

Monique Haicault

\author{
ERES | Empan »
}

2003/2 n ${ }^{\circ} 50 \mid$ pages 114 à 121

ISSN 1152-3336

ISBN 2-7492-0132-2

Article disponible en ligne à l'adresse :

http://www.cairn.info/revue-empan-2003-2-page-114.htm

\section{Pour citer cet article :}

Monique Haicault, « La dette dans les rapports de générations : une transmission en changement », Empan 2003/2 (nº50), p. 114-121.

DOI 10.3917/empa.050.0114

Distribution électronique Cairn.info pour ERES.

(C) ERES. Tous droits réservés pour tous pays.

La reproduction ou représentation de cet article, notamment par photocopie, n'est autorisée que dans les limites des conditions générales d'utilisation du site ou, le cas échéant, des conditions générales de la licence souscrite par votre établissement. Toute autre reproduction ou représentation, en tout ou partie, sous quelque forme et de quelque manière que ce soit, est interdite sauf accord préalable et écrit de l'éditeur, en dehors des cas prévus par la législation en vigueur en France. Il est précisé que son stockage dans une base de données est également interdit. 


\section{GÉNÉALOGIES ET HÉRITAGE, PARENTÉS ET RITUELS}

\section{La dette}

dans les rapports

de générations :

une transmission

en changement

Monique Haicault

La génération des Trente Glorieuses a été authentiquement politique, car plus que d'autres elle s'est impliquée dans les grands changements économiques, sociopolitiques et symboliques qui ont affecté les sociétés occidentales dans la seconde moitié du $\mathrm{XX}^{\mathrm{e}}$ siècle. On peut la considérer comme un observatoire vivant de la dynamique des changements dans les rapports sociaux de générations et de genre (Haicault, 1998).

Ouverts sur plus de démocratie, les changements sociaux ont affecté en profondeur les conditions matérielles de tous. Plus radicalement celles des femmes, par l'exercice libre d'une profession, l'autonomie économique et créatrice, l'accès libre à un logement et à l'espace public, le contrôle de la sexualité par la contraception, des modes de vie novateurs qui ont été doublés par une transformation plus novatrice encore des conditions immatérielles comme l'instruction libre et gratuite, avec l'accès aux professions, la participation à la vie politique et publique, les droits civiques et civils, de nouveaux modes de se penser, de se parler, de se représenter.

Des tensions entre hommes et femmes, certes, mais aussi entre générations ont secoué la famille. Les anciens systèmes de relations, les codes institués des échanges intrafamiliaux, de mère en fille, de père en fils et croisés, se sont déstabilisés. Qu'en est-il des solidarités au sein des familles agricoles et ouvrières qui servaient, hier encore, de protection à la menace de précarité et passaient pour immuables, tellement elles paraissaient naturelles (Schwartz, 1990)?

L'observation des pratiques intrafamiliales, principalement celles du « don contre-don », qui impliquent une sorte de dette entre personnes,

Monique Haicault, sociologue LEST, avenue Jules-Ferry, 13626 Aix-en-Provence cedex. Tél. 0442378500 .

E-mail haicault@univ-aix.fr http ://www.univ-aix.fr/lest

1. Il s'agit notamment d'une intervention orale de Pierre Bourdieu au GDr Famille du cNRS en 1993. 
met en relief de nouvelles configurations relationnelles. Les unes témoignent d'une opposition aux anciens modèles - encore vivaces - jusqu'à la rupture des liens de famille. Les autres tentent d'innover d'autres modes de relations en tenant à distance les injonctions des vieux modèles. Ces différentes manières de « contourner la dette » (Bloch et Buisson, 1991) entre générations témoignent d'un nouvel état des rapports sociaux inter et intrasexes, imbriqués à ceux de génération, dont les efforts de théorisation semblent en panne.

\section{La doxa familiale}

L'année 1993, année européenne de la solidarité entre générations, s'est accompagnée de nombreux travaux qui ont souligné la place des femmes au cœur de quatre générations, support en quelque sorte des liens familiaux horizontaux et verticaux.

L'exercice de la solidarité de la « génération pivot » des dernières décennies présente des traits dont le rappel permet de mieux saisir la nature des changements. Les femmes qui ont, aujourd'hui, entre cinquante et soixante ans y occupent une place décisive. Les aides prennent de multiples formes, matérielles et immatérielles, elles touchent aussi bien les ascendants que les descendants. Ces systèmes d'échanges sans contrat se rapprocheraient du rituel « don contre-don » des sociétés traditionnelles ; c'est dire leur poids dans le fonctionnement des relations familiales et dans la transmission d'un certain ordre social.

Elles relèvent d'un « allant de soi » des liens familiaux, d'une doxa familiale qui suppose : services, échanges, aides, assistances, mises à disposition, au nom de l'amour filial, au nom d'un imaginaire de la famille qui se veut unie, solidaire, coopérante, harmonieuse. Système de pensée, de règles et de comportements, la doxa de famille est puissante, au point que sa transgression menacerait les relations et l'équilibre affectif. Elle sert de jauge à l'évaluation de l'amour entre proches, à l'acquittement correct de la dette. Comme dans les sociétés traditionnelles, donc fermées au changement, le pouvoir des anciennes femmes sur les plus jeunes est ici quasi absolu pour assurer une transmission intacte. Les exemples ne manquent pas, y compris dans nos sociétés qui montrent par exemple ce qu'a été, et est encore, dans beaucoup de communautés culturelles sans long passé démocratique, la résistance des mères à « l'émancipation » de leur fille (Haicault, 2000).

La sociologie de la famille s'est souvent laissé prendre par une approche familialiste des rapports de générations, notamment pour tout ce qui concerne les liens familiaux - solidarités, « réseau familial utile », « économie solidaire »-, également pour la question de la transmission des règles. Ce « manque d'objectivation » dans la problématique et la conception de la famille a été souvent dénoncé par Pierre Bourdieu ${ }^{1}$.

\section{Les mères des solidarités familiales}

Comment se comportaient les anciennes mères de la génération pivot ? Le plus souvent invisibles, ces femmes étaient des grands-mères peu réclamantes dans des familles larges et cohabitantes de la France du milieu du siècle dernier. Elles acceptaient discrètement leur sort de dominées, cherchant à le transmettre tel quel à leur fille. Avec la conquête des droits civils et politiques, une nouvelle génération de femmes est née, une nouvelle génération de mères, de filles et de grands-mères. Des liens familiaux d'un autre type sont apparus. La dette de mère en fille a perdu de son évidence. Les aides reçues ou données sont devenues plus tangibles, jusqu'à un semblant de négociation. De nouvelles formes de transactions entre générations ébranlent ainsi, non sans heurts, les anciens modèles.

\section{Don et contre-don dans les échanges entre générations}

Les remarques qui suivent s'appuient sur des données recueillies auprès de femmes et d'hommes âgés de 50 à 68 ans, appartenant à plusieurs milieux sociaux et vivant le plus souvent en milieu urbain. Les femmes anciennes actives pour la plupart - ont toutes eu enfants et petits-enfants. Comme les hommes interviewés, elles ont souvent un père ou une mère encore en vie, parfois les deux. 
"On ne se parle plus»,

"Il ne mia pas

téléphoné depuis

troismois»
L'analyse s'attache aux contenus des relations entre quatre générations de femmes, étudiées sur plusieurs décennies. La génération des jeunes retraités est au carrefour des interactions. Elle entretient des liens avec ses parents âgés, des liens avec ses enfants - leurs filles correspondent aux femmes actives de mes recherches -, elle a aussi des relations avec ses petits-enfants - ceux dont j'ai étudié les modes précoces d'apprentissage des temps sociaux. Le regard se porte sur les relations qui se nouent dans les lignées directes de femmes et dans des lignées directes d'hommes, plus rarement dans le croisement des alliances. Deux types principaux de relations entre générations ont été dégagés des entretiens : les relations du don obligé, les relations du contournement de la dette.

\section{La dette des anciens modèles de transmission}

À partir des entretiens et des observations - étayées par d'autres travaux de chercheurs -, on note que " l'obligation de la dette » concerne davantage la fille que le fils. L'aide aux vieux parents repose sur les femmes sept fois sur dix (Cribier, 1985). De leur côté, Clément et Drulhe (1992) ont fait remarquer que les parents âgés ayant une fille, rentrent en maison de retraite six à huit ans plus tard que ceux qui ont un fils. Ce sont là de bons indicateurs statistiques du croisement entre rapports de génération et rapports de genre.

\section{La dette à la mère âgée, une relation silencieuse faite de souffrances}

La dette envers la mère âgée peut revêtir des formes tyranniques que les femmes rencontrées cachent le plus souvent, jusqu'à ce que leur corps cède et qu'elles tombent malades. Ce sont ces formes dont il est question essentiellement. Dans la plupart des cas, les relations s'alimentent de la culpabilité de la fille, savamment mais inconsciemment entretenue par la vieille mère qui rappelle au bon moment, en mère victime, l'obligation de la dette. Odile « doit» quotidiennement à sa mère plusieurs appels téléphoniques et plusieurs visites par semaine. Cette mère aisée, grande-bourgeoise, exerce sans le vouloir - et sans le voir - une véritable tyrannie sur cette belle femme, jeune grandmère qui, par contre, voit rarement sa petite-fille et sa propre fille, mariée en Italie, car elle « ne peut s'absenter plus de deux jours loin de sa mère ». Elle consacre beaucoup de son énergie et de son temps à cette mère possessive, à laquelle, depuis l'enfance, dire non a toujours été impensable. Odile a vécu en symbiose avec sa mère, en quête d'amour. Elle a reproduit une relation semblable avec sa propre fille. Celle-ci est partie au moment où sa santé commençait à lui signifier : danger. Éduquée au don, au dévouement, à la perfection du service, Odile mène une vie de femme autonome. Elle rencontre des hommes qui l'aiment, mais qui profitent aussi de ses qualités de femme oblative et supportent mal le partage avec la vieille mère. Actuellement enfermée dans cette relation sans contre-don, elle est entrée en dépression. 
Combien de femmes comme Odile, rencontrées ici et là, dans des milieux plutôt bourgeois, qui ne parviennent pas à transformer ce mode très policé de relations enraciné depuis l'enfance ! Certaines ont reconnu qu'elles ont parfois vécu ces rapports comme une corvée, mais n'ont jamais pensé le dire, ni même se l'avouer ou chercher des palliatifs. Il est vrai que les services humainement acceptables dans ces contextes sont encore rares.

Suzon, quant à elle, s'était mise dans l'obligation d'aller passer un week-end sur deux chez sa belle-mère, à cent kilomètres de là, son mari fatigué lui ayant laissé cette charge. Il l'avait assumée quelque temps, mais irrégulièrement, avec son frère : il se contentait de régler les affaires de bourse de la vieille dame, alors peu dépendante. Aujourd'hui âgée à son tour, Suzon, ayant vu mourir père, mari, belle-mère, s'étonne d'avoir pu subir une telle contrainte, sans aucune reconnaissance et en silence. Elle conclut par ces mots : "Il faut dire que c'était impossible dans cette famille, comme dans la mienne, de ne pas faire cela !». Depuis l'entretien, Suzon, qui a été opérée de deux cancers intestins et foie -, prend peu à peu conscience qu'elle est en train d' «exiger » de sa fille les mêmes services.

Dans un tel système de redevances, le stock affectif qui sous-tend le tout s'épuise vite. La vieille mère estime de son côté qu'elle ne reçoit pas assez d'amour et s'écrie : "On ne se parle plus », « Il ne m'a pas téléphoné depuis trois mois », «Je ne sais pas pourquoi », « Je m'endors et me répète, pourquoi ? pourquoi ? sans trouver de raison ». Quant aux enfants, ils estiment leurs efforts insuffisamment « reconnus ». Ils souhaiteraient vivre de telles relations comme un don et non comme un dû.

Cette génération est démunie, tiraillée entre la dette et la compassion pour les très vieux parents malades, seuls, dépendants, dégradés. Que faire ? Les abandonner, les prendre chez soi, les mettre en maison de retraite ? L'allongement forcé de la vie («forçonné », avoue l'une de ces femmes) est-il vraiment un bienfait quand il entraîne autant de souffrances ?

\section{La dette des fils à leur mère}

La doxa qui pèse sur les fils est beaucoup moins contraignante. Souvent, ils la déjouent en reproduisant un comportement semblable à celui des pères séparés à l'égard de leurs enfants, connoté d'oubli ou d'ajournement des tâches à remplir. S'ils refusent moins ostensiblement, ils se fâchent par contre moins souvent que les filles. Les formes dures de rupture des relations d'un fils avec une vieille mère sont plus rares. Elles sont vécues toutefois encore plus douloureusement, car ces femmes âgées, souvent veuves, déversent sur le fils un flot de fantasmes et d'attentes non comblées. Les tensions proviennent souvent des questions d'héritage ou bien des relations difficiles de la mère avec l'épouse du fils, nœud qu'il ne parvient pas facilement à desserrer. André dira : «On n'a plus rien à se dire avec ma mère, et d'ailleurs ma femme ne la supporte pas. »

Un mode de relation transmet ses tonalités les plus fines. Des positions de pouvoir générées puis transmises en famille créent ainsi des contenus de relations qui, faute d'avoir été repensées et exprimées, permettent en toute légitimité de donner ou de reprendre, de gratifier ou d'ignorer, d'être présent ou absent.

Ces transmissions reproduisent des configurations de liens familiaux dont la sociologie, avec la psychologie des généalogies, finira bien par dégager les fils et la trame. La socialisation d'une sorte de droit sur l'autre s'inscrit, pour le cas français, dans une longue tradition. Avec Napoléon, le droit sur la vie même de l'enfant n'at-il pas été maintenu comme un droit parental ? Le dogme de l'autorité parentale, exprimé par « Qui aime bien châtie bien », assorti de « C'est pour ton bien » (Miller, 1984), se retrouve dans beaucoup de comportements éducatifs, quand bien même ils ne seraient plus que verbaux. La loi du père, stigmatisée par un psychanalyste français comme dogme universel et naturel, est assortie d'une figure maternelle de femme pourvoyeuse, substitutive, toujours prête à « faire à la place » dès lors qu'elle tire son identité de l'illusion de son excellence ; deux imagos parentaux coriaces qui font système et nourrissent aussi les rapports de sexe. 


\section{Les échanges} se fout entre mières et filles plus quientre belles-mieres et belles-filles, ou qu'entre mières et fils ou gendres

\section{Les grands-mères, ressources du réseau familial utile}

Les observations portent ici sur les relations des personnes retraitées de moins de 70 ans avec leurs filles, leurs fils et leurs petits-enfants. Ces jeunes grands-mères et jeunes grands-pères sont sollicités cette fois par la demande des enfants, voire des petits-enfants. Quelles figures de relations enchevêtrées peut-on dégager des analyses ? Comment se manifeste l'ancien modèle de la dette obligée qui met en scène, cette fois, au moins trois générations ?

Rappelons qu'en France une femme sur deux devient grand-mère avant 52 ans, alors qu'elle a encore une belle carrière de femme devant elle puisque $91 \%$ des Françaises atteignent 60 ans sans problème ; leurs mères n'y parvenaient pas une fois sur deux (Desplanques, 1996). Une position stratégique unique dans l'histoire de l'humanité, qui concerne de toutes petites familles, de deux à quatre petits-enfants. Les échanges sont donc en principe plus simples, avec moins d'interactions potentielles. Cependant, ils paraissent plus compliqués, parfois douloureux eux aussi.

Les grands-mères ressources, nombreuses dans tous les pays anciennement industrialisés, sont fortement sollicitées par leurs filles actives pour alléger les gardes ou les suppléer quand les services publics font défaut. En France, il y aurait entre onze et treize millions de grands-mères, beaucoup sont ou ont été des grandsmères ressources.

Les échanges se font entre mères et filles plus qu'entre belles-mères et belles-filles, ou qu'entre mères et fils ou gendres. On reste dans le clan familial, puisqu'on transmet des pratiques semblables, des orientations éducatives similaires. Une recherche sur la prime éducation familiale a bien montré la force de cette transmission éducative de mère en fille (Haicault et Fouquet, 1992). Quand les orientations pédagogiques diffèrent, il s'ensuit souvent une distance, voire une rupture. En cas de garde irrégulière, la fille ou la belle-fille peut exiger que la grand-mère se déplace pour venir garder le jeune enfant chez lui.

Dans beaucoup de milieux observés, la garde est régulière et quasiment institutionnalisée. Le jeune enfant est souvent amené chez la grand-mère maternelle. Une famille rurale du sud-ouest de la France illustre cette situation relativement fréquente. À sa retraite, la mère est venue habiter tout près de sa fille. La petite fille, cinq ans, est portée encore endormie, très tôt chaque matin, chez sa grand-mère les horaires de la mère, aide-soignante, l'y obligent. L'enfant est alors recouchée avant d'être conduite à l'école par cette grand-mère qui la récupère le soir avant que les parents ne la reprenne. L'horizon de vie personnelle de la grand-mère est occupé par la régularité et l'allant de soi du service. Le propos ici n'est pas de savoir si chacun y trouve son compte, mais de saisir la force du lien qui crée de telles situations. 


\section{Des relations entre générations en train de basculer}

Des essais de négociations et de prise de conscience sont repérables, qui montrent que d'autres configurations relationnelles sont en train d'émerger. Cette novation ne semble pas tenir à la seule volonté des personnes, ni aux changements dans les modes de vie, ni au passage vers d'autres types d'affectivité, mais au moins à tout cela. Une autre étape des relations entre personnes, y compris familiales, est en train de naître, cherchant plus d'autonomie et d'engagement réfléchi.

\section{Un essai de négociation trop tardive}

L'analyse des bribes de l'histoire longue d'une ancienne professeure de français est éloquente. Mère de trois filles adultes, cette ancienne militante politique, féministe, écologiste instruite, est aussi artiste. Elle écrit et fait de la poterie. Divorcée depuis longtemps de son mari, ancien cadre, habitant la même grande ville, elle le voit toujours. Lui, semble par contre échapper aux relations complexes de ses filles et gendres avec leur mère. Judith s'est occupée depuis sa naissance d'un premier petit-fils qu'elle gardait le mercredi, l'emmenant vers ses lieux de loisir. Elle a transmis avec sensibilité et finesse le meilleur d'elle-même, ornant ses « dons » d'une ouverture sur la beauté du monde, de la terre, des textes, des valeurs, avec gaieté et joie du corps ; promenades ensemble à vélo dans la ville et à la campagne, initiation au film, à la photographie, à la pensée.

Plus tard, elle a fait de même pour la petite sœur, avec déjà moins de bonheur, car la relation à sa fille et à son gendre s'étant détériorée, la garde était devenue incertaine.

Clairvoyante, elle ne peut s'empêcher de faire sentir ce qui lui paraît « clocher» dans l'éducation donnée par le gendre avec la complicité silencieuse de sa fille. Mais, surtout, elle n'a jamais négocié ce qu'elle apportait, ou acceptait de donner, afin de mettre au clair l'absence évidente de contre-don. Le contre-don tacite étant les petits-enfants eux-mêmes, la fille «punissait » sa mère en la privant d'eux. Judith se confronte à un puissant modèle dont elle semble la seule à vouloir modifier les règles. Il est en effet porté et transmis par la très vieille mère espagnole, plaque tournante de toutes les informations, qui inculque ses propres valeurs par petites doses, au bon moment.

Des bouts d'entretien montrent le poids des doxas familiales et sur quel fonds de pouvoir et d'emprise psychologique se tissent des rapports d'appropriation réciproques, constitutifs souvent des sagas générationnelles.

« Ma mère est légitimée par les voies plurielles de la tribu, celles qui n'étaient pas dans son idéologie ont été rejetées, moi, mon frère, ma sœur. L'exclusion de mon frère l'a conduit à son cancer, à la mort. "Il a payé sa discorde avec la famille", disait ma mère. Selon elle, moi aussi je paye en plein. J'ai compris ça, aussi je ne parle plus de mes problèmes de tremblements, je le cache quand je vais la voir.

" Ma mère faisait régner une sorte de terreur par la crainte du châtiment. Elle considère comme secte tout ce qui sort de l'église de son village espagnol. Mes enfants me font payer le fait que j'ai échappé à la tribu, que j'ai divorcé. Pour eux, les gens qui divorcent sont forcément coupables. »

L'amour de Judith pour ses petits-enfants, la joie qu'elle tire de ce qu'elle transmet sont pris en otage par ses filles et ses gendres. La relation de service dans laquelle elle s'est mise fait qu'elle ne peut plus rien négocier, tout étant considéré comme dû dès lors qu'elle serait payée du seul fait de garder les enfants. La menace de la priver d'eux est constante mais jamais vraiment exprimée. Cela est plus fréquent qu'on ne le croit. Parfois, c'est le fils qui va priver sa mère du petitenfant, ou qui va la tenir à distance, sans que jamais les choses ne soient abordées clairement. Chacun préfère taire ce qui le fait souffrir, comme s'il fallait à tout prix préserver l'imaginaire de la « famille heureuse ».

À côté de ces femmes qui tentent, un peu tard dans leur vie, de sortir du non-dit des relations, d'autres se tournent hardiment vers la recherche d'une « voie du milieu ». 


\section{Le don contre-don négocié : une autre figure des relations entre générations}

Une autre figure de relation avec les parents âgés et avec les enfants et petits-enfants émerge des entretiens et des observations. Elle vise à établir des contrats, à négocier les liens, la dette, à affirmer l'autonomie de chacun. Elle suppose un travail sur soi, conscient, qui, bien que rare, semble annonciateur de changements sociaux débordant le champ des relations entre personnes.

Déjà jeune grand-mère, cette femme actuellement thérapeute, cultivée et fine, raconte comment elle a évolué dans sa relation à son père, alors âgé de 81 ans.

« Aujourd'hui, il est moins sur ses starting blocks qu'avant, en tout cas

Il faut faire éclater toutes

ces histoires de famille, comme celles des nations, aller au-delà il n'est plus dans la revendication, la quête. Il appelle tous les quinze jours, avant que je vienne déjeuner, et me demande ce qui me ferait plaisir ; il me signifie ainsi qu'il m'attend, je sais que cela lui fait plaisir. Je ne vis plus les choses comme avant, ce que je n'aime pas en lui ne m'affecte plus, sa raideur, son intimidation. Il m'arrivait de pleurer en rentrant, en me disant: "Il ne reconnaîtra jamais sa dureté, son manque de souplesse", j'aurais voulu qu'il évolue. Maintenant je le vois, mais ça ne m'agace plus, je laisse venir, je vois de mieux en mieux que les autres sont un miroir, je regarde plus sereinement les autres et moi-même, ce qui fait que la vie est moins bêtement répétitive, on a l'impression de moins récidiver, car je mets plus de conscience dans tout ce que je vis. »

Elle a su trouver un mode relationnel, entre la soumission à l'obligation de la dette et la violence du rejet. Son attitude est le fruit d'une réflexion assidue, personnelle, qui lui a permis de conserver son autonomie tout en enrichissant la relation à son père et, par suite, avec son fils, ses petits-enfants et même avec les hommes.

Ces nouvelles femmes «travaillent » leur relation aux enfants, aux petits-enfants, cela éclaire leur vie, enrichit l'entourage, car tout est lié. L'idée de la nécessité de la parole, de l'écoute au bon moment, leur paraît essentiel pour maintenir de bonnes relations familiales. Écoute du cœur, secret de la bonne relation installée dans une temporalité plus souple, dosage subtil de ce qu'il faut dire et ne pas dire, car " on raconte trop en famille ». Une relation imprégnée du respect de l'autre affirme sa nouveauté par son refus d'un droit sur l'autre, tel que la famille l'exprime dans son langage le plus ordinaire - « mon enfant, mon mari, ma femme, ma mère, etc. » - et qui bien souvent exerce ce droit que les jeunes générations rejettent à leur manière.

Une sophrologue qui rencontre beaucoup de femmes en recherche explique son travail dans ce domaine : "Se désengager ne veut pas dire indifférence ou abandon, mais le moyen d'établir de nouveaux rapports. Se désengager, c'est certes savoir dire non sans animosité, à ma mère si elle abuse, à ma fille si elle m'instrumentalise. C'est aussi, 
dans notre ressenti, quitter la rancœur pour trouver plus de liberté et de douceur intérieure. C'est très difficile, tout un travail que je fais sur moi-même, seule et avec d'autres, mais qui retentit sur mes relations de proximité affective et familiale. »

Y a-t-il, du côté des hommes, des changements aussi forts dans les relations entre générations ? Des grands-pères de la « voie du milieu » existent probablement qui savent contourner les contraintes sans les rejeter, ni les nier, qui savent établir des relations plus déliées avec leurs enfants et petits-enfants, des relations inventives. Le poids des doxas est peut-être moins lourd sur eux que sur les femmes, elles qui sont au cœur de la transmission des règles de l'énorme sphère de la reproduction sociale. J'ai rencontré des couples grand-père / petit-fils dans les jardins publics, ou pêchant ensemble ou bien marchant dans la montagne. S'il y a transmission, elle semble plus technique et instrumentale que relationnelle, un champ de recherche à continuer d'explorer.

Pour finir, donnons la parole à un expert en relations, grand-père lui-même, qui proclame de manière optimiste l'avenir d'une solidarité extrafamiliale généralisée :

«Il faut faire éclater toutes ces histoires de famille, comme celles des nations, aller au-delà, mettre l'accent sur l'humanité UNE et ne plus parler d'égalité dans la famille, car c'est sur d'autres bases qu'il faut penser, ne plus se limiter à son moi d'abord, ma famille d'abord, mon pays d'abord. Il faut repenser en termes de globalité. Dans nos réactions, on réagit chacun dans son précarré, il faudrait apprendre à mieux s'écouter et à se parler. Voir aussi dans les attitudes de l'autre quelque chose qui doit retenir l'attention et non pas se sentir visé, car victime ou coupable, c'est pareil. Les autres parlent toujours avec leurs symptômes, leurs réactions. Écouter, car le propre du fonctionnement familial, comme en politique, repose sur la non-écoute de l'autre, on regarde la télé, on écoute des bêtises, mais pas assez nos enfants et nos parents. »

\section{Conclusion}

Les héritages symboliques sont de bons témoins de l'état des rapports sociaux entre générations. Contradictoires, faits d'alliances, de solidarité, de soumission, de révoltes, de pouvoir, ces rapports sociaux sont invisibilisés dans les enchevêtrements des relations affectives familiales sous le poids des modèles toujours prêts à renaître.

Les figures traditionnelles de la dette obligée et consentie s'appuyaient sur des rapports intrafamiliaux, relevant de modèles qui se sont transmis de mère en fille, de mère en fils.

Des mouvements secouent les relations sur trois ou quatre générations, non sans douleur, non sans heurts. De nouvelles figures de liens familiaux, plus rares et en recherche, pourraient annoncer d'autres manières de vivre les relations familiales et, plus généralement, les relations entre personnes devenues autonomes, ouvertes à l'écoute et capables alors de négociation.

\section{Bibliographie}

Bloch, F. ; Buisson, M. 1991. « Du don à la dette, la construction du lien social familial », Revue $d u$ MAUSS, $\mathrm{n}^{\circ} 11$, Paris, La Découverte.

Clément, S. ; Drulhe, M. 1992. « De l'offre rationalisée à une demande polymorphe », Vieillir dans la ville, Paris, L'Harmattan.

Cribier, F. (dir.). 1985. Pénélope : Vieillesses des femmes, Paris, ministère des Droits des femmes.

DesPlanques, G. 1996. « La situation familiale des personnes âgées », Données sociales, INSEE, Paris.

Haicault, M. ; Fouquet, A. 1992. Apprentissage des temps sociaux, l'héritage du quotidien, INSEE, CNRS, LEST.

Haicault, M. 1998. « Les jeunes retraités, une génération intervalle dans le temps et l'espace urbain », revue Prévenir, Vieillir, n 35, p. 123-130.

HaICAUlt, M. 2000. L'expérience sociale du quotidien, corps, espace, temps, Presses de l'Université d'Ottawa, coll. «Théorie sociale ».

MiLler, A. 1984. C'est pour ton bien, racines de la violence dans l'éducation de l'enfant, Aubier.

SCHWARTZ, O. 1990. Le monde privé des ouvriers, hommes et femmes du Nord, Paris, PUF. 